\title{
FIGO Stage IA2
}

National Cancer Institute

\section{Source}

National Cancer Institute. FIGO Stage IA2. NCI Thesaurus. Code C96247.

A FIGO stage term that applies to gynecologic cancers. For cervical cancer, it refers to invasive cancer that is diagnosed microscopically only. The stromal invasion is more than $3.00 \mathrm{~mm}$ but no more than $5.00 \mathrm{~mm}$ with a horizontal spread of $7.00 \mathrm{~mm}$ or less; there is no FIGO stage A2 for endometrial cancer. 